\title{
Learning olfactory codes using matrix factorization on 2DG uptake patterns from rats
}

\author{
Amir Madany Mamlouk \\ From 1st International Workshop on Odor Spaces \\ Hannover, Germany. 4-7 September 2013
}

Recent studies based on both the analysis of odor descriptors [1] and psychophysical studies [2] have suggested that olfactory perception is mainly dominated by odor pleasantness. In contrast, findings on the sensory side [3] as well as on the perceptual side [4] indicate an odor perception space that is spanned by about 30 dimensions. On the way to understand the basic principles in the decoding of olfactory information from the single molecular compounds, we are focusing in this study on the first level of information processing in the sense of smell: The olfactory bulb (OB). We know that the olfactory epithelium is separated into four regions [5] of olfactory receptor protein expression and we expect a similar organization in the olfactory bulb [6]. With 2-DG-uptake-images, Johnson et al. [7] measured odorant-specific activity patterns, covering $98 \%$ of the bulbar surface with a resolution of one glomerulus per pixel. Using a database of 143 such bulbar activity fingerprints, we propose the so called non-negative matrix factorization [8] to decompose the data into a set of common features. These features can be best described as the coding alphabet while the uptake images of single molecular compounds resemble natural occurring code words. Interestingly, we can achieve three meaningful alphabets with a different size: 4,6 , and 9 digits. Only the last one is suitable for actually coding the compounds, as a single compound is evoking activity of only about $1 / 8$ of the bulbar surface, with an average code word length of two to three digits. The other two decompositions might reflect the superglomerular organization of the receptor neurons in the bulb, like e.g. the above mentioned expression patterns and neuronal subnetworks that directly result from the targeting mechanisms guiding the neurons from the $\mathrm{OE}$ to the $\mathrm{OB}$. We argue that the proposed decompositions are not only reflecting the chemotopic organization of the bulbus but rather giving a first hint on the coding scheme underlying olfactory perception.

Published: 16 April 2014

\section{References}

1. Madany Mamlouk A, Chee-Ruiter C, Hofmann UG, Bower JM: Quantifying olfactory perception: mapping olfactory perception space by using multidimensional scaling and self-organizing maps. Neurocomputing 2003, 52-54:591-597.

2. Khan RM, Luk C-H, Flinker A, Aggarwal A, Lapid H, Haddad R, Sobel N: Predicting odor pleasantness from odorant structure: pleasantness as a reflection of the physical world. J Neurosci 2007, 27:10015-23.

3. Haddad R, Khan R, Takahashi Y, Mori K, Harel D, Sobel N: A metric for odorant comparison. Nat Methods 2008, 5:425-429.

4. Madany Mamlouk A, Martinetz T: On the dimensions of the olfactory perception space. Neurocomputing 2004, 58-60:1019-1025.

5. Ressler KJ, Sullivan SL, Buck LB: A zonal organization of odorant receptor gene expression in the olfactory epithelium. Cell 1993, 73:597-609.

6. Mori K, Takahashi YK, Igarashi KM, Yamaguchi M: Maps of odorant molecular features in the Mammalian olfactory bulb. Physiol Rev 2006, 86:409-33.

7. Johnson Ba, Woo CC, Leon M: Spatial coding of odorant features in the glomerular layer of the rat olfactory bulb. J Comp Neurol 1998, 393:457-71.

8. Lee DD, Seung HS: Learning the parts of objects by non-negative matrix factorization. Nature 1999, 401:788-91.

\section{doi:10.1186/2044-7248-3-S1-O3}

Cite this article as: Mamlouk: Learning olfactory codes using matrix factorization on 2DG uptake patterns from rats. Flavour 2014 3(Suppl 1): O3. 